
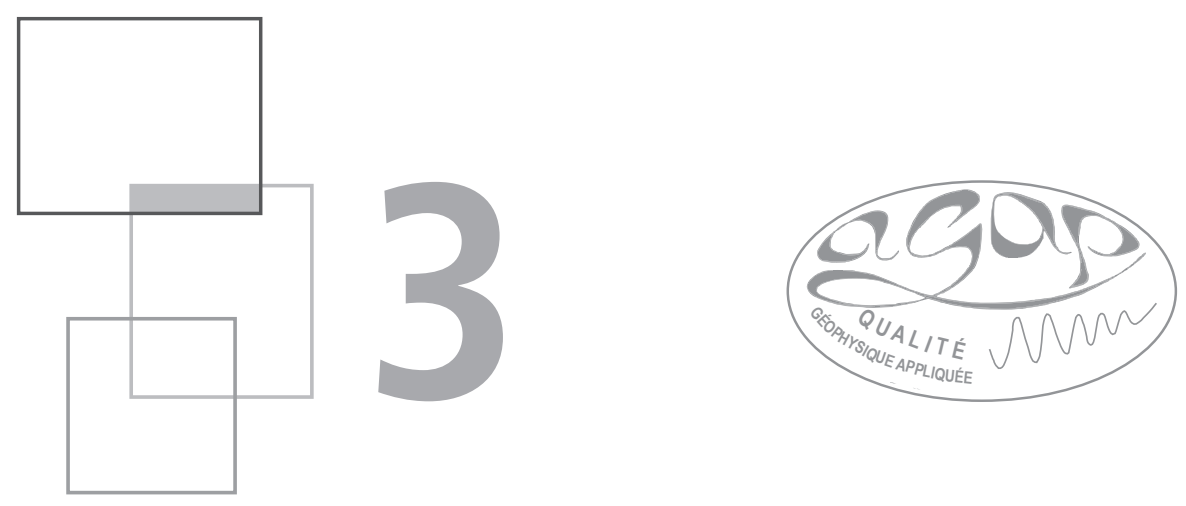

\title{
Diagraphie acoustique
}

\author{
J.-L. Mari, C. Vergniault, F. Coppens
}

\subsection{Introduction}

Depuis de nombreuses années, la transmission d'une onde acoustique à travers les milieux est utilisée dans la mesure en forage. La diagraphie acoustique est une diagraphie visant à mesurer la vitesse de propagation du son dans les formations géologiques, en utilisant un outil acoustique composé d'un système émetteur et d'un système récepteur. À l'origine, cette mesure, appelée sonique (sonic log) était essentiellement destinée à mesurer l'intervalle $(\Delta \mathrm{t})$ des temps d'arrivée de la première onde de compression, au niveau de deux récepteurs, distants de 25 à 50 centimètres, l'énergie étant émise par un émetteur situé à environ 1 mètre du premier récepteur. On obtient une courbe de lenteur (inverse de la vitesse) de laquelle est déduite la vitesse de propagation de l'onde sonique réfractée (15 à $30 \mathrm{kHz})$ dans les formations. Dans ce cas, on ne prend en compte que le pointé de la première arrivée de

Ce chapitre de l'ouvrage Sismique en forage et diagraphies acoustiques est publié en Open Access sous licence creative commons CC-BY-NC-ND permettant l'utilisation non commerciale, la distribution, la reproduction du texte, sur n'importe quel support, à condition de citer la source.

(C) EDP Sciences, 2018

DOI: $10.1051 / 978-2-7598-2262-1 . c 005$ 
l'onde de compression $(\mathrm{P})$ des milieux traversés. L'utilisation de la diagraphie dite sonique pour déterminer la vitesse des ondes de compression est une pratique courante et relativement ancienne (Summers et Broding, 1952 ; Vogel, 1952).

La diagraphie acoustique en champ total (full wave form acoustic logging) ou carottage acoustique repose sur l'analyse et le traitement des différents trains d'onde (ondes réfractées, ondes guidées, ondes réfléchies) enregistrés par l'outil.

Les enregistrements du champ d'ondes total permettent de déterminer les vitesses de propagation des différents modes et certains paramètres pétrophysiques, et d'obtenir des informations lithologiques et mécaniques (Gaudiani, 1982 ; Arditty, Garens et Staron, 1984 ; Morris, Little et Letton, 1984 ; Paillet et Turpening, 1984 ; Mari, Coppens, Gavin et Wicquart, 1992 ; Mari, Arens, Chapellier et Gaudiani, 1998 ; Mari, Gaudiani et Delay, 2011).

Le forage peut être un trou ouvert, un trou tubé (acier et/ou PVC), un trou tubé cimenté. Dans ce dernier cas, la diagraphie acoustique est utilisée pour faire un contrôle de cimentation et pour déterminer les paramètres caractéristiques des formations (vitesses...).

La diagraphie acoustique a une résolution verticale de quelques centimètres et une investigation latérale centimétrique pour les modes d'interface, décimétrique à métrique pour les modes rétractés, et de la dizaine de mètres pour les modes réfléchis. Elle fournit au puits une information de détail, fonction de la profondeur, sur les vitesses des ondes acoustiques et les caractéristiques pétrophysiques des roches. L'exploitation des modes réfléchis peut conduire à une image comparable à une section micro-sismique en temps qui, dans les cas favorables, permet de suivre les limites de couches et d'en estimer le pendage. Combinée à une diagraphie de densité, elle fournit un log d'impédance acoustique, fonction de la profondeur, qui est transformé en fonction du temps après correction, calibration et calage.

La figure 3.1 est un exemple de diagraphie acoustique en champ total. L'outil acoustique (partie gauche de la figure) est un outil souple, de petit diamètre, composé d'un émetteur et de deux récepteurs. La distance entre l'émetteur et le premier récepteur est de $3 \mathrm{~m}$, la distance entre les deux récepteurs est de $25 \mathrm{~cm}$. La référence de profondeur est le sol. La cote profondeur correspond à la profondeur du point situé à mi-distance entre les 2 récepteurs. La partie droite de la figure est un exemple de section acoustique obtenue en utilisant un couple émetteur-récepteur, distant de $3 \mathrm{~m}$. Dans cette représentation, l'axe vertical représente la profondeur à laquelle est situé le capteur (ici $3 \mathrm{~m}$ ), l'axe horizontal représente le temps d'écoute (ici $3 \mathrm{~ms}$ ). La section acoustique est composée de traces acoustiques. Chaque trace acoustique est l'enregistrement acoustique mesuré par le récepteur, distant de $3 \mathrm{~m}$ de l'émetteur, sur une durée d'écoute de $3 \mathrm{~ms}$. Sur l'enregistrement, on peut identifier différents trains d'onde. 

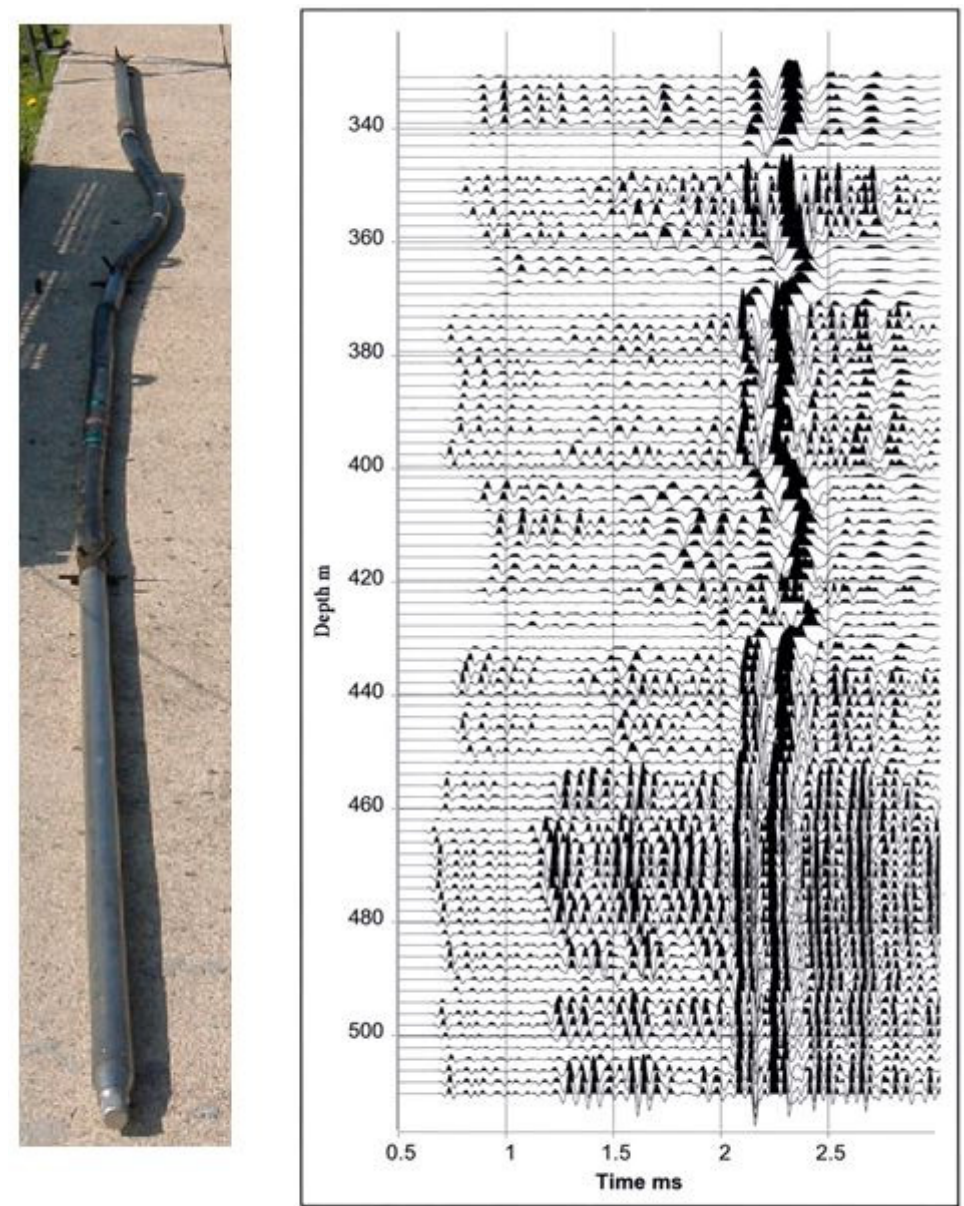

Figure 3.1 Diagraphie acoustique en champ total - outil et section acoustique.

\subsection{Acquisition des données de diagraphies acoustiques}

Dans ce paragraphe, nous présentons successivement :

- les moyens nécessaires à l'acquisition des données ;

- la mise en œuvre sur le terrain. 


\subsubsection{Moyens nécessaires à l'acquisition des données}

Les moyens concernent : les équipements, les véhicules et le personnel.

\section{Équipements}

1. une unité de diagraphie comprenant : un certain nombre de sondes, un treuil sur lequel s'enroule un câble de plusieurs centaines de mètres auquel sont connectées les sondes, une unité d'enregistrement et de numérisation (la numérisation pouvant être faite au niveau des sondes), un système de visualisation et d'impression des enregistrements terrain, un système de mesure de la cote profondeur ;

2. une ou plusieurs sondes acoustiques ;

3. une source sismique (chute de poids), si une opération PSV est prévue avec la diagraphie acoustique ;

4. un système de levage avec poulies pour la descente des sondes de diagraphie (et de PSV).

Les équipements sont vérifiés (maintenance, étalonnage) périodiquement.

\section{Véhicules}

1. soit : une unité de logging (de préférence tout-terrain/tout-chemin), permettant le transport du personnel et des équipements (sondes de diagraphies et sonde PSV en option...) ;

2. soit : une unité de logging et un véhicule ou une remorque permettant le transport de la source PSV (si une opération PSV est prévue avec la diagraphie acoustique).

\section{Personnel et compétences}

1. deux opérateurs qualifiés pour la mise en œuvre (treuil, descente des sondes de diagraphie, acquisition) ;

2. un géophysicien (chef de mission) qualifié pour le contrôle qualité des données à l'acquisition et qui peut être aussi opérateur.

\subsubsection{Mise en œuvre sur le terrain}

Dans cette partie, nous décrivons successivement :

\subsubsection{Déroulement d'une opération de diagraphie acoustique en puits vertical}

En puits vertical, on fait l'hypothèse de symétrie cylindrique des formations géologiques par rapport à l'axe du puits. Pour cette mesure, le trou doit être en eau 
(boue de forage). La sonde acoustique est descendue centrée, par des centreurs. Il est recommandé de faire au préalable une mesure des diamètres du forage (diamétreur).

Après avoir effectué le calage du zéro (référence sonde) à un plan de référence (radier, table de rotation...) ou au sol, la sonde est descendue à une profondeur donnée choisie par l'opérateur pour faire des mesures en stationnaire. Ces mesures permettent de vérifier la qualité des enregistrements acoustiques, le bon fonctionnement de l'outil, la répétitivité des mesures, d'évaluer le rapport signal sur bruit et de régler certains paramètres d'acquisition (gains...). L'outil est ensuite descendu en fond de puits. Une acquisition de contrôle peut être faite à la descente, pour s'assurer qu'il n'y aura pas de saturation à l'acquisition. L'opération de mesure est ensuite faite à la remontée à vitesse constante en fonction du pas d'échantillonnage en profondeur. Une vitesse de remontée de 4 à $6 \mathrm{~m} / \mathrm{min}$ est classique.

\subsubsection{Sondes acoustiques}

Les outils utilisés sont des outils de type monopôle ou de type dipôle. Les outils monopôles sont les plus utilisés. Les émetteurs et les récepteurs sont multidirectionnels (figure 3.2a). Les émetteurs génèrent dans le fluide une onde de compression qui donne naissance dans la formation à une onde de compression (onde $\mathrm{P}$ ) et une onde de cisaillement (onde $S$ ) aux angles limites de la réfraction. Les outils acoustiques de type dipôle sont utilisés pour accéder aux paramètres $S$ des formations lentes et sont équipés d'émetteurs et de récepteurs polarisés. De tels outils génèrent des ondes de compression polarisées perpendiculairement à l'axe du forage. Ces ondes de compression créent à la paroi du puits des modes de flexure qui donnent naissance dans la formation à des pseudo-ondes de cisaillement se propageant parallèlement à l'axe du puits (figure $3.2 \mathrm{~b}$ ).

A

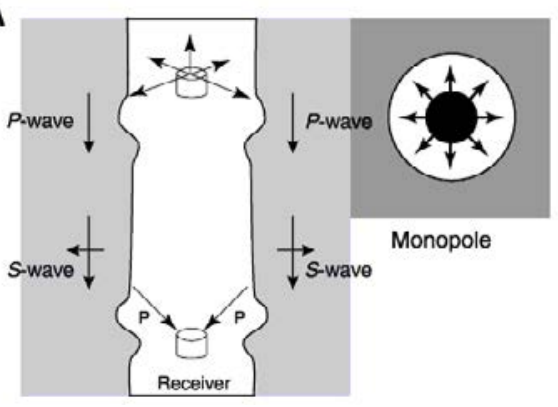

B

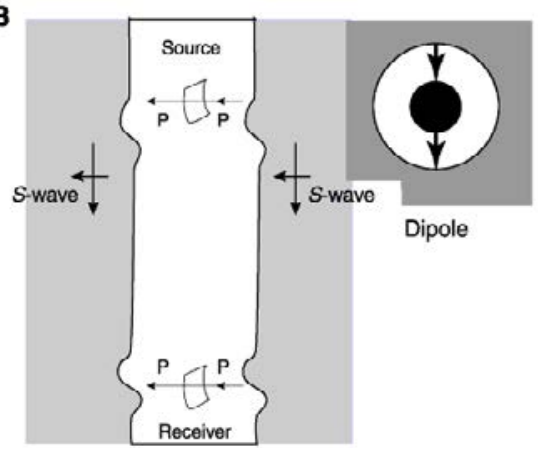

Figure 3.2 Types d'émetteurs Array sonique. a : monopole émetteur donnant une impulsion multidirectionnelle. $b$ : dipôle émetteur donnant une impulsion dirigée. (Modifié de Zemanek et al., 1991.) 
Lorsque la vitesse de cisaillement de la formation est inférieure à la vitesse $\mathrm{P}$ du fluide de forage, l'onde de flexion se déplace à la vitesse des ondes $S$ et c'est donc le moyen diagraphique le plus fiable pour estimer un log de vitesse de cisaillement. Le problème est que ces outils du domaine pétrolier sont rigides et de grande longueur (environ $10 \mathrm{~m}$ de long et $10 \mathrm{~cm}$ de diamètre). Leur mise en œuvre n'est pas adaptée aux forages géotechniques. En revanche, il existe une adaptation pour le domaine de la géotechnique, le PSSL (PS Suspension Logging) qui est souple. La méthode du PSSL a été initialement développée dans le milieu des années 1970 par des chercheurs de l'Oyo Corporation du Japon (Kaneko et al., 1990) avec des géophones comme récepteur. Aujourd'hui, la société Robertson Geologing et la société Geovista ont également développé une sonde avec des hydrophones. L'espacement entre les sources et les récepteurs est de 2 à $3 \mathrm{~m}$ et la plage des fréquences couvre le domaine de 100 à $1000 \mathrm{~Hz}$. La source est un électroaimant horizontal qui produit une onde de pression dans le fluide du trou de forage (source électrodynamique). À la paroi du forage, cette onde de pression est convertie en ondes sismiques $\mathrm{P}$ et $\mathrm{S}$, qui se déplacent radialement à partir de la paroi du trou. Ces ondes sont reconverties en ondes de pression dans le fluide du trou de forage et détectés par les récepteurs (composante verticale pour l'onde P; composante horizontale pour l'onde S).

Un outil acoustique est caractérisé par :

- le type de fonctionnement :

- monopôle : fréquence d'émission 10-40 kHz,

- dipôle : fréquence d'émission $1-3 \mathrm{kHz}$;

- le type d'émetteur et de récepteur :

- magnétostrictif,

- piézo-électrique;

- le nombre d'émetteurs et de récepteurs :

- classique avec un ou deux émetteurs et deux récepteurs,

- antenne réceptrice avec de quatre à huit récepteurs ;

- l'écartement entre récepteurs : de dix à cinquante centimètres ;

- le déport de la source par rapport au premier récepteur : de un à cinq mètres ;

- les caractéristiques mécaniques :

- ossature rigide ouvragée,

- ossature souple ;

- le pas d'échantillonnage en temps :

- 5 ou 10 us pour un outil monopôle,

- $20 \mu$ s pour un outil dipôle ;

- la durée d'écoute :

- 2 ou $5 \mathrm{~ms}$ pour l'analyse des modes réfractés,

- $10 \mathrm{~ms}$ ou plus pour l'analyse des modes réfléchis.

La figure 3.1 gauche est un outil acoustique, souple, de petit diamètre $(50 \mathrm{~mm})$, de type monopôle, qui est utilisé pour des études en forage géotechnique mais aussi 
pour des mesures acoustiques dans des puits de type pétrolier. L'émetteur est magnétostrictif (fréquences d'émission : 17-22 kHz). Il peut être équipé de deux couples de récepteurs (récepteurs proches, 1-1,25 m; et récepteurs lointains, 3-3,25 m). Les exemples acoustiques présentés au chapitre 5 ont été obtenus avec cet outil, développé par P. Gaudiani.

La figure 3.3 montre des exemples d'outils acoustiques.

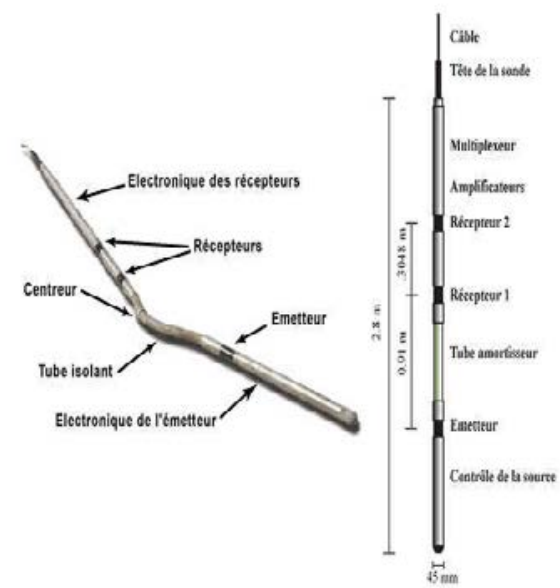

a

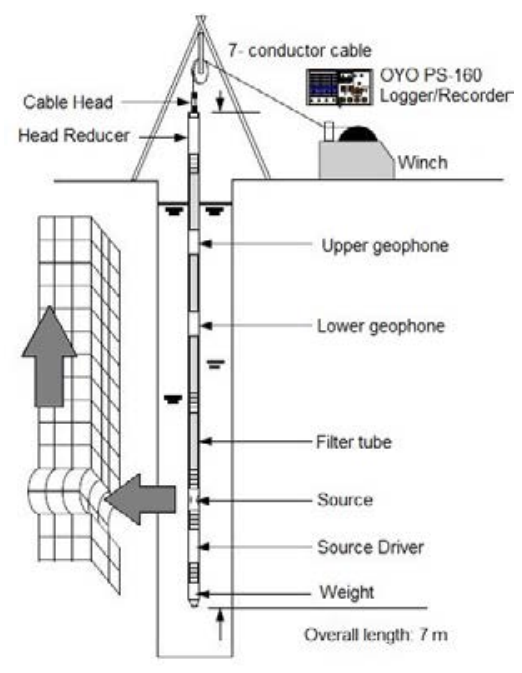

C
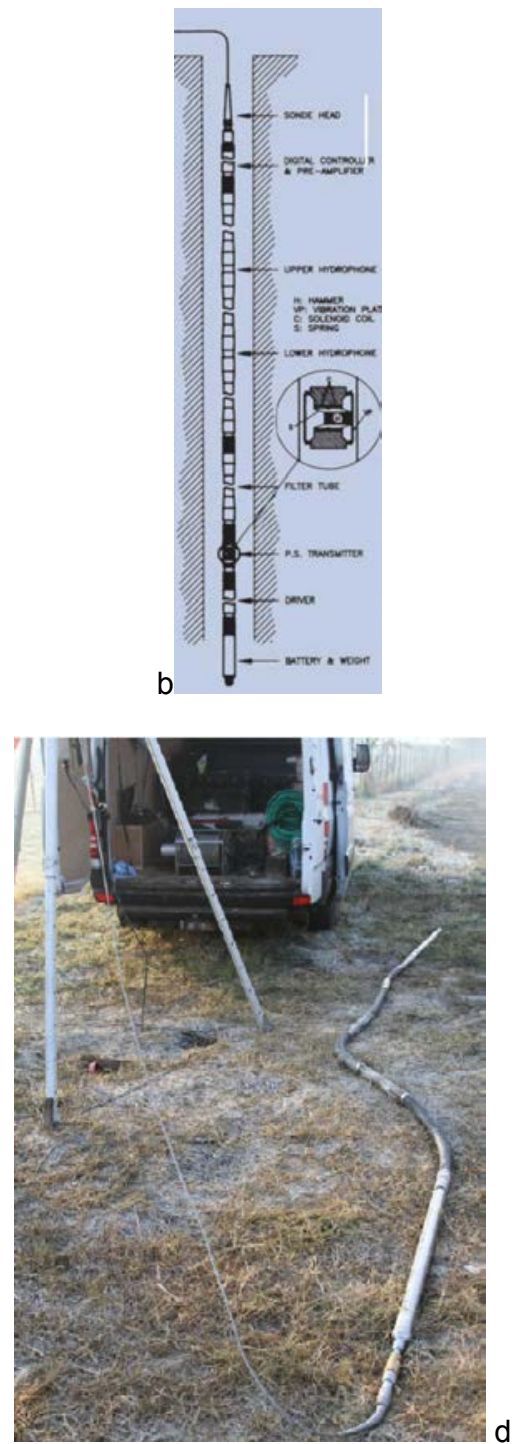

Figure 3.3 Outils acoustiques. a : outil monopôle (Mount Soppris). b : outil dipôle (Robertson). c et d : PSSL (OYO) principe et vue de l'outil (document : GeoVision-EDF). 


\subsubsection{Paramètres d'acquisition et de visualisation}

L'enregistrement acoustique peut être visualisé sous forme de sections acoustiques à déport constant (iso-déport), le déport étant la distance séparant l'émetteur d'un récepteur. Chaque section iso-déport est un enregistrement à deux dimensions (temps : axe vertical - profondeur ou longueur filée : axe horizontal, ou inversement). Le pas d'échantillonnage en profondeur doit être choisi pour éviter le phénomène d'aliasing spatial sur les sections iso-déport en vue d'un traitement ultérieur des données acoustiques.

En pratique, le pas d'échantillonnage en profondeur est choisi égal à une fraction de la distance séparant deux récepteurs de l'outil, ce qui permet pour les arrivées réfractées de simuler des tirs directs - inverses et de faire des mesures de vitesse compensées.

Les gains d'acquisition doivent être choisis pour éviter toute saturation, notamment pour les modes guidés. Cependant, si l'acquisition est faite pour obtenir un log de vitesse en onde $\mathrm{P}$, on peut choisir des gains pour amplifier les ondes de compression de façon à faciliter le pointé du temps de première arrivée (pointé par seuil), quitte à saturer les modes guidés et à faire un second " run " pour acquérir les modes guidés en amplitude préservée.

\subsubsection{Diagraphie acoustique en puits dévié}

Si le puits est dévié, les ondes réfléchies aux limites des couches traversées par le forage sont enregistrées par l'outil acoustique. Ces ondes pourront être exploitées et traitées pour fournir une micro-sismique de détail au voisinage du puits.

\subsubsection{Sécurité}

La sécurité du chantier doit être assurée par le chef de mission, en accord avec le Système Qualité du prestataire. Les accès à la zone de mesures seront sécurisés.

\subsubsection{Contrôle Qualité}

Lors de la remontée de la sonde acoustique, l'opérateur contrôle la qualité des enregistrements sur les différents récepteurs de la sonde acoustique, notamment le niveau de bruit. Il peut être utile de réduire la vitesse de remontée, en zone bruitée, le pas d'échantillonnage en profondeur devant rester constant.

\subsubsection{Production}

En moyenne, pour une diagraphie acoustique, la vitesse de logging est de 4 à $6 \mathrm{~m} /$ min. Cette diagraphie est rarement effectuée seule. Elle s'inscrit dans un ensemble de mesures obtenues dans plusieurs " runs". 


\subsection{Ondes acoustiques}

Dans un puits vertical, les outils monopoles permettent d'enregistrer cinq modes de propagation :

- l'onde de compression réfractée ;

- l'onde de cisaillement réfractée, uniquement en formation rapide $\left(V_{S}>V_{P}\right.$ fluide) ;

- l'onde de fluide ;

- deux modes guidés dispersifs qui sont les ondes de pseudo-Rayleigh et les ondes de Stoneley :

- les ondes de pseudo-Rayleigh sont des ondes coniques réfléchies (Biot, 1956), dispersives dont les vitesses de phase et de groupe approchent aux basses fréquences $(<5 \mathrm{kHz})$ la vitesse $S$ de la formation, et asymptotiquement aux hautes fréquences $(>25 \mathrm{kHz}$ ) la vitesse de propagation de l'onde de compression dans le fluide. Ces ondes n'existent qu'en formations rapides ;

- les ondes de Stoneley sont des ondes dispersives d'interface qui, en formation rapide, ont des vitesses de phase et de groupe qui approchent asymptotiquement et par valeur inférieure aux hautes fréquences la vitesse du fluide et qui, en formation lente, sont plus dispersives et plus sensibles aux paramètres des ondes $\mathrm{S}$ de la formation. Aux basses fréquences, les ondes de Stoneley sont analogues aux ondes de tube observées en downhole (chapitre 1) et PSV (chapitre 2).

Les mesures acoustiques réalisées en champ total sont représentées sous forme de sections iso-déport ou de collections en point émetteur commun ou point récepteur commun, analogues à celles utilisées en sismique. Une section iso-déport ou carottage acoustique est un ensemble d'enregistrements acoustiques représentés en fonction de la profondeur, obtenus avec une distance émetteur-récepteur fixe.

Nous présentons maintenant un ensemble de collections en point émetteur commun et un ensemble de sections iso-déport montrant les différents types d'ondes que l'on peut observer sur ces enregistrements. Les collections en point émetteur commun sont des sismogrammes synthétiques qui ont été réalisés en utilisant les programmes de modélisation de Jacques Quiblier (1997). Ses programmes permettent la modélisation de données acoustiques en formations lentes ou rapides. La formation est infinie, élastique et isotrope. Elle est définie par des vitesses de propagation des ondes $\mathrm{P}\left(\mathrm{V}_{\mathrm{p}}\right)$ et des ondes $\mathrm{S}\left(\mathrm{V}_{\mathrm{s}}\right)$, par la densité $\rho$, et deux facteurs de qualité $(\mathrm{Qp}, \mathrm{Qs})$ caractéristiques de l'atténuation. Le puits de diamètre constant $(16,1 \mathrm{~cm})$ rempli d'eau $\left(\mathrm{V}_{\mathrm{f}}=1500 \mathrm{~m} / \mathrm{s}, \rho \mathrm{f}=1 \mathrm{~g} / \mathrm{cm}^{3}\right)$ est de longueur infinie. L'outil de longueur infinie a les propriétés acoustiques du fluide. Il est composé d'un émetteur (monopôle ou dipôle) et de neuf récepteurs (points de mesure). La distance émetteur - premier récepteur est de $1 \mathrm{~m}$. La distance entre 2 récepteurs consécutifs est de $12,5 \mathrm{~cm}$. Le pas d'échantillonnage en temps est de $10 \mu \mathrm{s}$, la durée d'écoute de $4 \mathrm{~ms}$. En chaque point de mesure, l'algorithme calcule les trois composantes du 
déplacement (Ur, U $\theta, \mathrm{Uz}$ : déplacements radial, tangentiel et vertical) et la pression P. Dans nos simulations, les caractéristiques des formations sont :

- $\quad$ pour la formation lente $\left(\mathrm{V}_{\mathrm{s}}<\right.$ Vitesse du fluide dans le puits) :

$\mathrm{V}_{\mathrm{p}}=2760 \mathrm{~m} / \mathrm{s}$,

$\mathrm{V}_{\mathrm{s}}=1380 \mathrm{~m} / \mathrm{s}$,

$\rho=2,3 \mathrm{~g} / \mathrm{cm}^{3}$,

$\mathrm{Qp}=\mathrm{Qs}=90$;

- $\quad$ pour la formation rapide $\left(\mathrm{V}_{\mathrm{s}}>\mathrm{Vitesse}\right.$ du fluide dans le puits) :

$\mathrm{V}_{\mathrm{p}}=4000 \mathrm{~m} / \mathrm{s}$,

$\mathrm{V}_{\mathrm{s}}=2000 \mathrm{~m} / \mathrm{s}$,

$\rho=2,3 \mathrm{~g} / \mathrm{cm}^{3}$,

$\mathrm{Qp}=\mathrm{Q} s=90$.

La figure 3.4a montre la collection point-émetteur commun obtenue avec un outil monopôle en formation lente. Sans amplification, la seule onde visible est l'onde de Stoneley. Avec une amplification de $60 \mathrm{~dB}$, l'onde réfractée P apparaît clairement. La mesure de sa pente permet la détermination de la vitesse $V_{p}$ du milieu.

La figure 3.4b montre la collection point émetteur-commun obtenue avec un outil dipôle en formation lente. Sans amplification, seul le mode flexural est visible entre 2 et 3,5 ms. Après amplification, l'onde réfractée P apparaît entre 1 et 1,5 ms. Le mode flexural donne une très bonne estimation de la vitesse $\mathrm{Vs}$ de la formation.

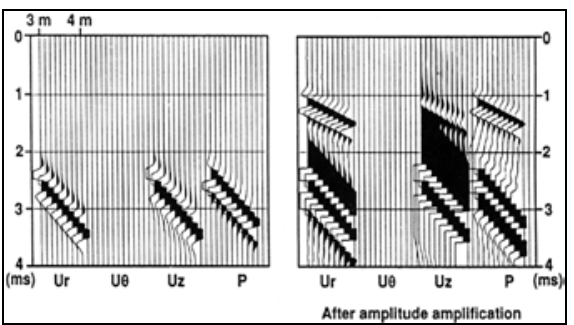

a

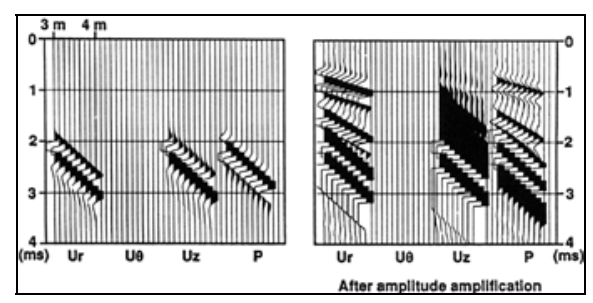

C

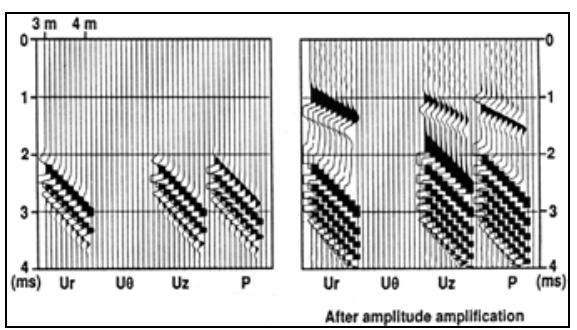

b

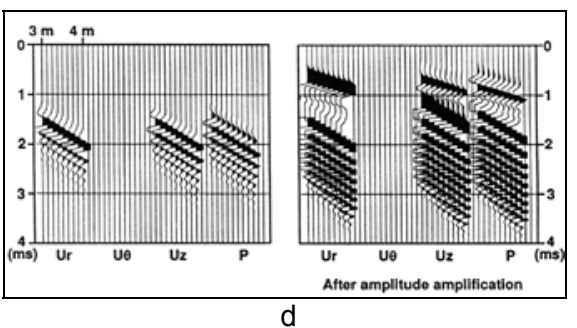

Figure 3.4 Diagraphie acoustique en formations lente et rapide. a : outil monopôle en formation lente. $b$ : outil dipôle en formation lente. $c$ : outil monopôle en formation rapide. $d$ : outil dipôle en formation rapide. 
La figure $3.4 \mathrm{c}$ montre la collection point-émetteur commun obtenue avec un outil monopôle en formation rapide. Après amplification, l'onde réfractée $\mathrm{P}$ apparaît dans l'intervalle 0,5-1 ms, l'onde réfractée $S$ dans l'intervalle 1,5-2 ms et l'onde de Stoneley, clairement visible sans amplification, dans l'intervalle 2-3,5 ms.

La figure $3.4 \mathrm{~d}$ montre la collection point-émetteur commun obtenue avec un outil dipôle en formation rapide. Le mode flexural très énergétique est présent dans l'intervalle 1,5-2 ms, le mode réfracté $\mathrm{P}$ dans l'intervalle 0,5-1 ms après amplification.

La figure 3.5a est un exemple de section iso-déport de $3 \mathrm{~m}$, correspondant donc à des mesures sur un récepteur distant de $3 \mathrm{~m}$ de l'émetteur, où ces propagations sont clairement visibles. La source est un émetteur magnétostrictif de type monopôle. La présence de l'onde réfractée $S$ indique que la formation est rapide. Lorsqu'il y a un contraste d'impédance entre deux formations dont le pendage est proche de celui du plan perpendiculaire à l'axe du trou, ces différentes ondes (réfractions et ondes d'interface) peuvent se convertir et se réfléchir comme le montre la figure $3.5 \mathrm{~b}$ et faire apparaitre sur les sections iso-déport des événements en chevron à vitesse apparente lente. De telles réflexions peuvent également se produire aux raccords de cuvelage, en présence de zones cavées ou de toute autre hétérogénéité de paroi de puits.

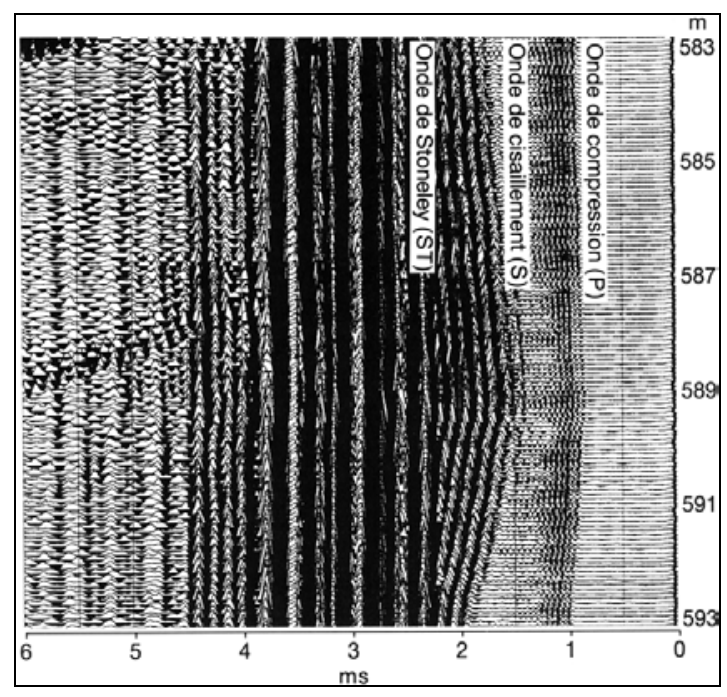

a

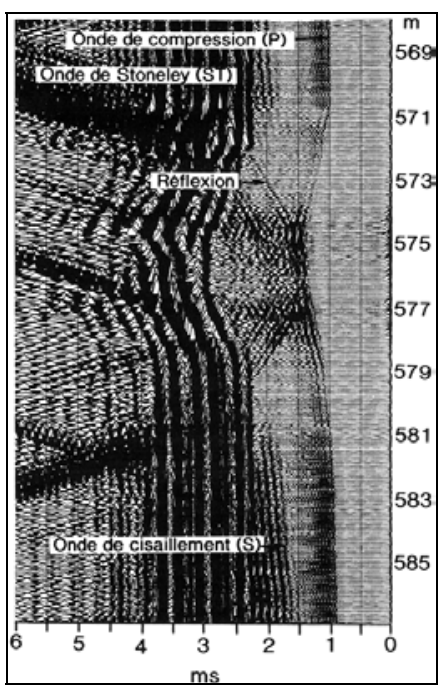

b

Figure 3.5 Sections acoustiques (document SEMM). a : exemple de section iso-déport obtenue avec une source monopôle. On observe l'arrivée $P$ réfractée, I'arrivée $S$ réfractée et l'onde de Stoneley. $b$ : exemple d'ondes $P$ réfractées-réfléchies et d'ondes de Stoneley réfléchies lors de contrastes d'impédance dans le puits. Sur une section iso-déport, ces arrivées se présentent sous la forme caractéristique d'événements en chevron, à vitesse apparente lente. 


\subsection{Séquence de traitement}

Le traitement classique d'une diagraphie acoustique permet d'obtenir la relation temps-profondeur et les logs de vitesse au puits et certains paramètres mécaniques tels que le coefficient de Poisson.

La séquence de traitement comprend :

1. Édition (élimination des enregistrements de mauvaise qualité).

2. Calcul des vitesses acoustiques par pointé des temps d'arrivée des différents trains d'onde ou par balayage en vitesse et semblance.

3. Contrôle qualité des vitesses (mesure du coefficient de corrélation) et des pointés (par exemple par mise à plat du train d'onde par application de corrections statiques égales aux temps pointés).

Remarques :

- Si l'algorithme de pointé utilise un seuil, la détection des pointés erronés (spikes et sauts de cycle) doit être lors de l'édition des logs de vitesse. Cette technique est uniquement applicable aux ondes de compression.

- Si les vitesses sont mesurées par semblance, il est recommandé d'utiliser un outil avec déport important entre l'émetteur et le premier récepteur (de l'ordre de 2 à $3 \mathrm{~m}$ ) et ayant au minimum 4 récepteurs. La mesure est facilitée si les trains d'ondes sont bien séparés en temps.

En option :

1. Mesure des amplitudes des différents trains d'onde et calcul des logs d'amplitude et atténuation.

2. Mesure des fréquences des différents trains d'onde et calcul des logs de fréquence (atténuation, résolution...).

3. Calcul de la porosité acoustique (formule de Wyllie).

4. Calcul de films synthétiques. Il est recommandé d'effectuer le calage (méthodes Block shift et $\Delta \mathrm{t}$ minimum) des mesures acoustiques $\Delta \mathrm{t}$ sur les mesures PSV.

5. Calcul des modules élastiques (géo-mécanique : choix des modèles utilisés).

6. Traitement de type "sismique réflexion " des ondes réfléchies et obtention des sections micro-sismiques au voisinage du puits (puits déviés ou horizontaux).

On compte un jour à plusieurs jours (voire semaines : un traitement de microsismique est équivalent à un traitement de sismique réflexion) de traitement, en fonction des options de traitement demandées. Si le traitement demandé est uniquement le log de vitesse $\mathrm{P}$, pointé par seuil, il peut être obtenu en temps réel sur le terrain.

Nous présentons des exemples de traitement classique de données acoustiques.

Le premier exemple (Mari et al., 2011, figure 3.6) est un exemple de données acoustiques acquises en formation rapide $\left(V_{S}\right.$ de formation $>V_{P}$ du fluide de puits). 

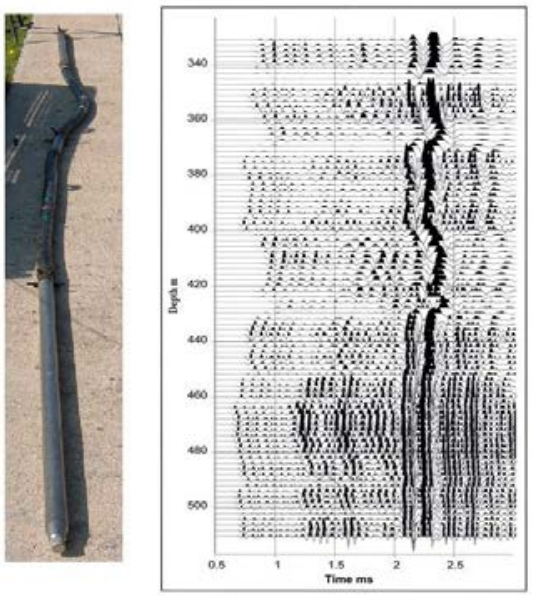

Mari et al., 2011

a

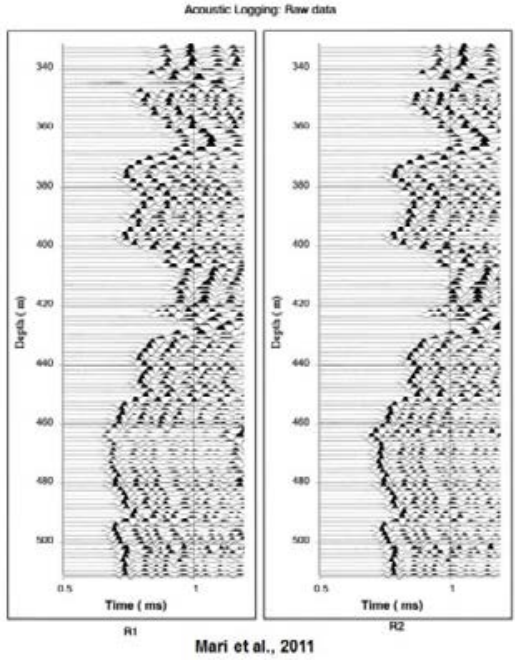

b

Figure 3.6 a et b Exemple d'enregistrement de données acoustiques.

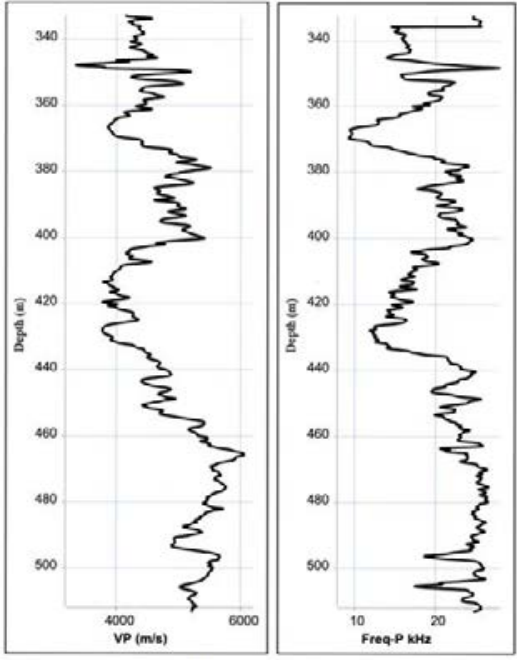

Mari et al., 2011
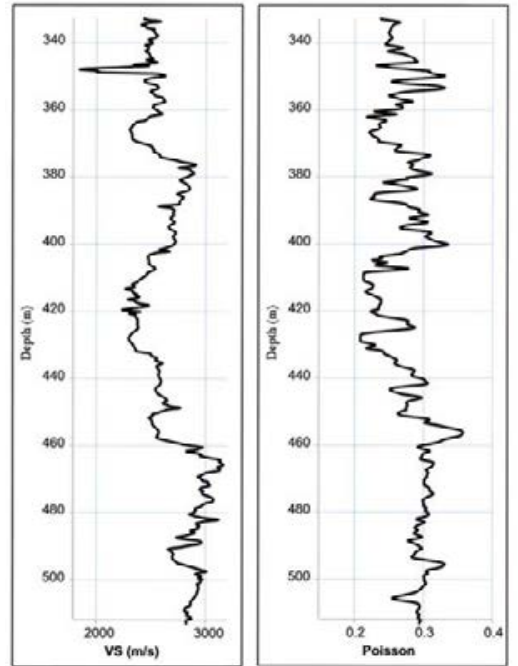

Mari et al., 2011

Figure $3.6 \mathrm{c}$ et d Exemple de traitement de données acoustiques - mesure des vitesses. $a$ : outil et section acoustique iso-déport $3 \mathrm{~m} . \quad b$ : enregistrements acoustiques sur 2 récepteurs séparés de $25 \mathrm{~cm}$. c : logs de vitesse de compression et de fréquence. $d$ : logs de vitesse de cisaillement et coefficient de Poisson. 
Sur la section acoustique iso-déport $3 \mathrm{~m}$ (figure 3.6a), on peut voir successivement l'onde réfractée $P$ en première arrivée, l'onde réfractée $S$ et les ondes de Rayleigh associées dans l'intervalle temps 1-2 ms, ces dernières étant particulièrement bien visibles pour des profondeurs supérieures à $440 \mathrm{~m}$. La figure 3.6b montre les deux sections acoustiques iso-déport $3 \mathrm{~m}$ et $3,25 \mathrm{~m}$, dans l'intervalle temps 0,5-1,1 ms, centré sur l'onde réfractée P. Les ondes acoustiques de type Stoneley sont visibles au-delà de $2 \mathrm{~ms}$. On peut noter que ces dernières ont un contenu fréquentiel plus élevé pour des profondeurs supérieures à $440 \mathrm{~m}$. Le traitement a consisté à calculer les logs de vitesse $V_{\mathrm{P}}$ et $\mathrm{V}_{S}$, le log de coefficient de Poisson et le log de fréquence de l'onde réfractée $\mathrm{P}$ en utilisant la fréquence instantanée. Les différents logs sont présentés en figure 3.6c et 6d.

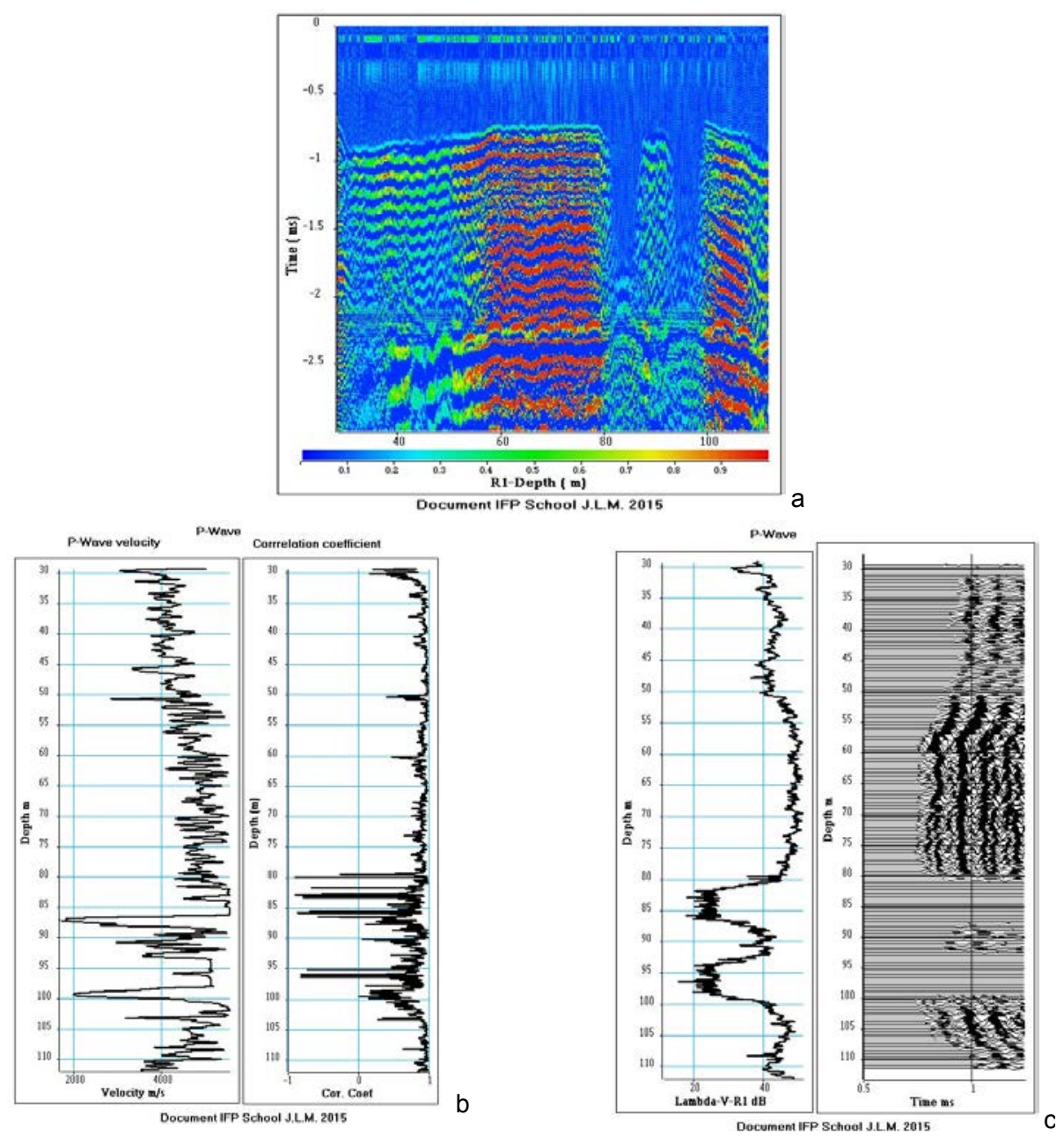

Figure 3.7 Exemple de traitement de données acoustiques. a : section acoustique isodéport $3 \mathrm{~m} . \mathrm{b}$ : log de vitesse (ondes) et log de corrélation. Les faibles valeurs de vitesse et du coefficient de corrélation indiquent la présence de niveaux karstiques. c : log d'amplitude des ondes P. Les très faibles amplitudes indiquent la présence de niveaux karstiques. 
Les données acoustiques du second exemple (Mari et Porel, 2015) ont été acquises dans une formation carbonatée. Les résultats sont présentés en figure 3.7. La figure $3.7 \mathrm{a}$ montre la section acoustique iso-déport $3 \mathrm{~m}$, on peut voir successivement l'onde réfractée $\mathrm{P}$, l'onde réfractée $\mathrm{S}$ et les ondes de Rayleigh associées, l'onde de fluide très haute fréquence à vitesse apparente infinie, située juste avant l'onde de Stoneley. On peut noter dans l'intervalle de profondeurs $80-100 \mathrm{~m}$, la présence de deux zones où les ondes sont fortement atténuées. La figure 3.7b montre le log de vitesse de l'onde réfractée $P$ et le log de corrélation associé, utilisé pour contrôle qualité de la mesure de vitesse. Dans les zones à vitesse lente et à forte atténuation, ce coefficient est faible. La figure 3.7c montre le log d'amplitude de l'onde P, obtenu par méthode SVD (décomposition en valeur singulière), dans le même intervalle de profondeurs. Dans cet intervalle, on peut noter une faible vitesse de formation, une forte atténuation, un faible coefficient de corrélation qui indiquent la présence de niveaux karstiques. Ces données font partie de l'étude de cas qui sera présentée au chapitre 5 .

\subsection{Imagerie acoustique}

Dans cette partie, nous décrivons simplement les procédés d'imagerie par réfraction et par réflexion.

\subsubsection{Imagerie acoustique par réfraction}

Le temps de transit total $T_{i, j}$ entre un émetteur $i$ et récepteur $j$ d'une onde réfractée est égal à la somme des délais à l'aplomb du point émetteur $\left(D_{i}\right)$ et du point récepteur $\left(D_{j}\right)$ augmenté du temps de transit associé au réfracteur $\left(X_{i, j} / V\right.$ avec $X_{i, j}$ distance entre l'émetteur $i$ et le récepteur $j, V$ vitesse du réfracteur) :

$$
T_{i, j}=\frac{X_{i, j}}{V}+D_{i}+D_{j}
$$

avec,

$$
\frac{X_{i, j}}{V}=\sum_{k=i}^{j-1} \mathrm{~d} t_{k, k+1}
$$

La quantité $\mathrm{d} t_{k, k+1}$ est le temps de propagation dans la formation entre deux positions successives de profondeur $k$ et $k+1$. La figure 3.8 schématise le trajet de l'onde réfractée entre une source $S$ à la position $i$ et un récepteur $\mathrm{R}$ à la position $j$. Les délais $\mathrm{D}$ sont des fonctions simples des paramètres de la boue (épaisseur et vitesse $\mathrm{hm}$ et $\mathrm{Vm}$ ) et des paramètres de la zone altérée de puits (épaisseur et vitesse ha et $\mathrm{Va}$ ). Le délai en $\mathrm{S}$ est égal au temps de trajet entre $\mathrm{S}$ et $\mathrm{B}$ moins le temps de trajet entre $\mathrm{A}$ et $\mathrm{B}$. Le calcul du délai à chaque cote profondeur permet d'estimer l'extension de la zone altérée de puits. Ce paramètre n’est en général jamais mesuré. 
Si la vitesse de la zone altérée de puits est supérieure à la vitesse de la formation $(\mathrm{Va}>\mathrm{V})$, la mesure de lenteur par la méthode acoustique ne conduit pas à estimer la vitesse de la formation, mais celle de la zone altérée de puits.

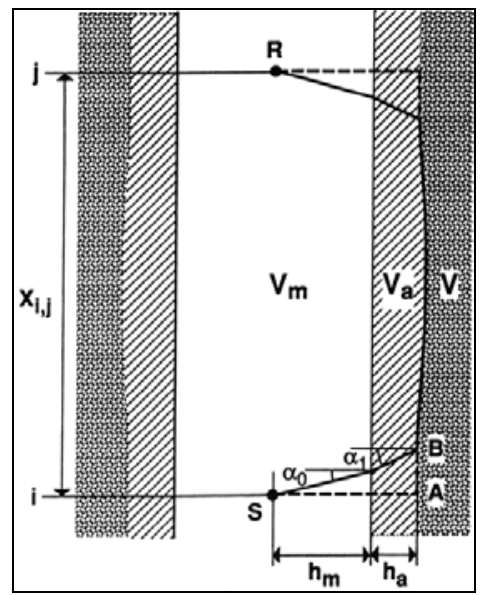

Figure 3.8 Schéma du trajet de la première arrivée réfractée (d'après Coppens et Mari, 1995).

La lenteur moyenne de propagation d'une onde sur un intervalle de profondeur donné correspond au retard acquis par l'onde sur cet intervalle. Pour une même position en profondeur de l'émetteur (réciproquement récepteur), le retard peut être mesuré en faisant la différence de temps d'arrivée de l'onde sur chacune des positions récepteurs (réciproquement émetteurs) situés dans l'intervalle de profondeur. De ce fait, la lenteur d'une formation peut être estimée en mesurant le retard de l'onde en se servant des enregistrements soniques triés, soit en collection point émetteur commun en profondeur, soit en collection point récepteur commun en profondeur. La moyenne des deux retards forme alors une lenteur compensée des effets de puits.

Les méthodes de mesure de lenteur basées sur le pointé des temps de première arrivée pour chaque trace fournissent des logs avec une résolution égale à la distance séparant deux récepteurs (entre un demi-pied et deux pieds). La méthode la plus connue est la méthode de pointé par seuil d'énergie minimum. Si le seuil a été mal choisi, ou si des problèmes d'atténuation ou de bruit modifient les énergies, il peut se produire des sauts de phase de une ou plusieurs périodes (dits sauts de cycle), faussant les mesures des temps.

Avec les outils à champ total, il est possible de contrôler la qualité du pointé et d'éviter les sauts de phase. Le pointé par seuil peut alors être avantageusement remplacé par des techniques de pointé qui prennent en compte d'autres critères pour suivre une onde. Mari et al. (1992) ont proposé une méthode de pointé basée sur l'utilisation de techniques issues de l'intelligence artificielle pour le suivi d'une onde particulière d'une trace à l'autre et d'un point de tir à l'autre. Le suivi de l'onde est réalisé en utilisant des critères de forme et de continuité. La forme de l'onde est 
définie par son amplitude et sa fréquence apparente. La continuité est exprimée par une faible variation de forme et par une faible différence de temps de l'onde d'une cote à l'autre. L'utilisation d'une telle méthode permet d'assurer un pointé cohérent en temps, amplitude et fréquence. La géométrie de l'outil (nombre de récepteurs et distance entre récepteurs) et la géométrie d'acquisition (pas d'avancement) permettent une redondance d'information à chaque cote profondeur et conduisent pour chaque paramètre mesuré à estimer une valeur de dispersion ou d'erreur.

La figure 3.9 montre huit sections iso-déports (de 9 à 12,5ft) enregistrées avec un outil multi-récepteurs. Les huit sections sont présentées sur une fenêtre temporelle de $1 \mathrm{~ms}$ encadrant la première arrivée. Les temps de pointé des premières arrivées $\mathrm{P}$ ont servi à calculer le log de lenteur et son log de dispersion de mesure (fig. 3.10a et b).

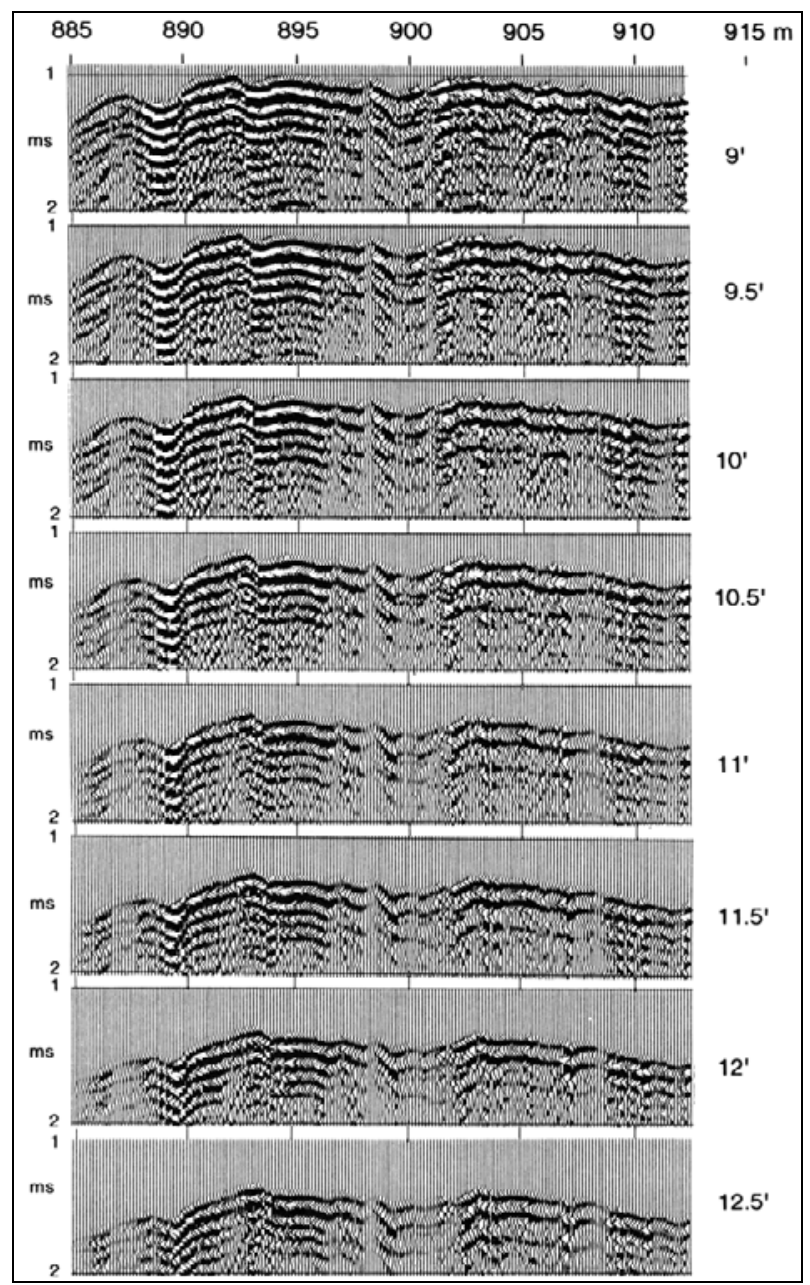

Figure 3.9 Sections iso-déports allant de 9 à 12,5 pieds, enregistrées avec un outil multirécepteur (d'après Mari et al., 1992). 


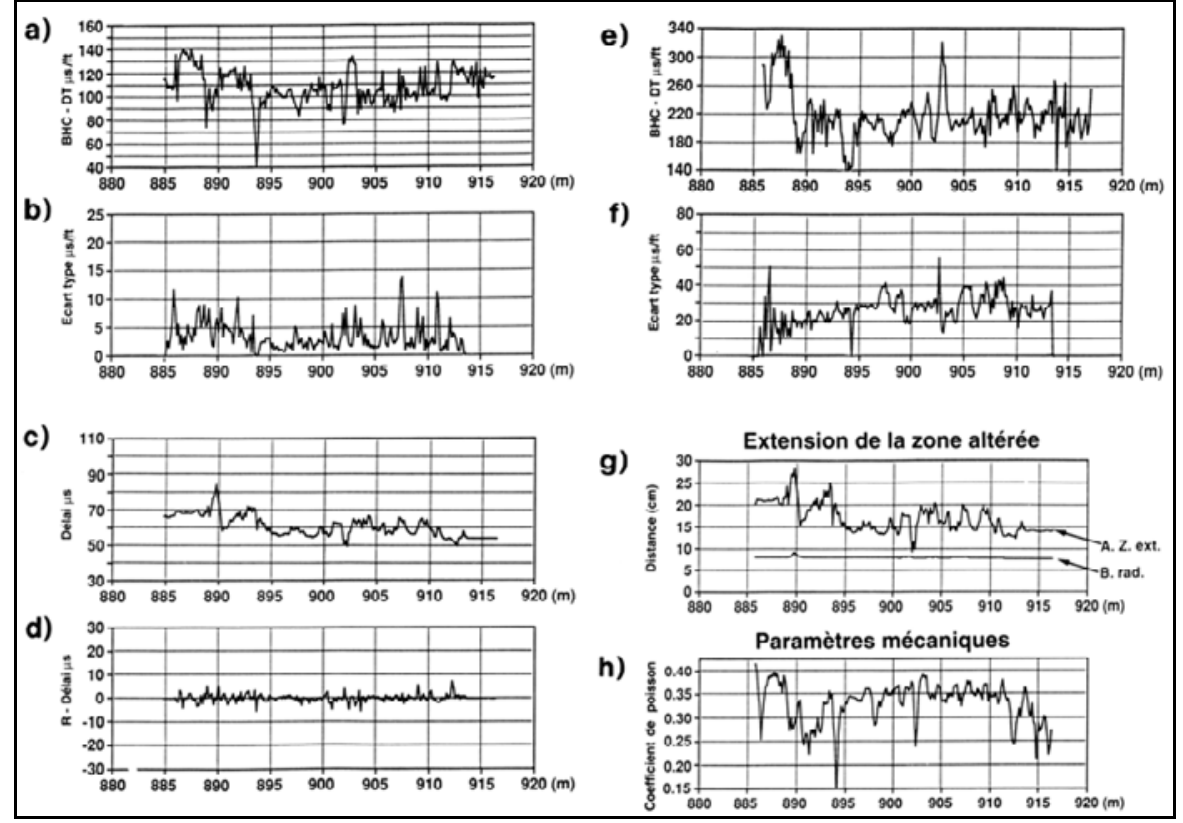

Figure 3.10 Exemple de logs acoustiques en réservoir gréseux (d'après Coppens et Mari, 1995). Outil monopôle (onde P) : a : lenteur ; $b$ : écart type sur la lenteur ; $c$ : délai ; : délai résiduel. Outil dipôle (onde de flexion \# onde $S$ ) : e : lenteur ; $f$ : écart type sur la lenteur ; $g$ : extension de la zone altérée (onde $P$ ) ; $h$ : coefficient de Poisson.

Les lenteurs étant connues, il est alors possible de calculer le log des délais (fig. 3.10c). Les temps théoriques d'arrivée de l'onde réfractée sont ensuite calculés pour chaque tir à partir des logs de lenteur et de délai. La différence entre les temps calculés et les temps réels représente l'erreur de dispersion de mesure. Elle est présentée comme un $\log$ de délai résiduel (fig. 3.10d) qui n'excède pas $5 \mu$ s soit un demi-pas d'échantillonnage en temps dans l'exemple étudié.

La formation étudiée étant une formation argilo-gréseuse lente (absence de modes réfractés $S$ sur les sections monopôles), la lenteur $S$ de la formation a été obtenue à l'aide des modes de flexion générés par un outil de type dipôle. La mesure des temps d'arrivée de l'onde de flexion directe donne accès au log de lenteur $S$ et à son log d'écart type associé (fig. 3.10 et 3.10f). La combinaison des logs de lenteur P et S conduit au coefficient de Poisson (fig. 3.10h).

La mesure des temps d'arrivée de l'onde réfractée P pour chaque couple émetteur récepteur sur l'ensemble du puits donne accès au délai en tout point de mesure et donc à une image de l'extension de la zone altérée de puits. La figure $3.10 \mathrm{~g}$ montre le log d'extension de zone altérée obtenue dans la formation argilo-gréseuse. 
Cet exemple fait ressortir que la profondeur d'investigation du mode réfracté est de quelques dizaines de centimètres. Par rapport à la paroi du puits, la zone altérée atteint localement des épaisseurs de $20 \mathrm{~cm}$. Les augmentations de zone altérée dans les zones poreuses (890-899 m ; 911-916 m) sont corrélées à la présence de formations peu consolidées. Dans les zones argileuses (899-911 m), les augmentations de la zone altérée sont corrélées à l'argilosité.

Cet exemple montre que la diagraphie acoustique à champ total fournit non seulement la lenteur des formations mais également une imagerie au voisinage du puits avec investigation latérale décimétrique à métrique pour les modes réfractés. Nous montrerons que l'analyse des modes réfléchis et diffractés permet d'étendre le pouvoir d'investigation de la diagraphie acoustique.

\subsubsection{Imagerie acoustique par réflexion}

Le traitement des modes réfléchis conduit à l'obtention de sections acoustiques à très haute résolution (quelques dizaines de centimètres) fournissant une image avec une investigation de quelques mètres par rapport à l'axe du puits.

En diagraphie acoustique en champ total, le document le plus facilement accessible est la section acoustique à déport constant (iso-déport). Les figures 3.11 et 3.12 montrent les résultats de travaux expérimentaux réalisés dans une carrière (Mari et al., 1994). La figure 3.11 (partie haute) montre la coupe géologique établie à partir des informations de puits (puits vertical noté R1 et puits fortement dévié). Le puits dévié est foré dans la couche d'oolithe blanche de $80 \mathrm{~m}$ d'épaisseur. La tête de puits est la cote de référence des abscisses. Pour les ordonnées, les marqueurs géologiques sont repérés en profondeur par rapport au toit de la carrière. À l'abscisse du puits vertical, le puits dévié est à $40 \mathrm{~m}$ de profondeur. La section acoustique à déport constant (figure 3.11, partie basse) montre deux types d'événements : les événements isochrones à vitesse apparente très grande et les événements obliques. Les événements à vitesse apparente grande sont les arrivées réfractées, les modes d'interface (ondes de pseudo-Rayleigh et ondes de Stoneley) et les arrivées réfléchies sur des marqueurs acoustiques parallèles au drain. Les événements obliques sont des arrivées réfléchies sur fractures pseudo-verticales ou sur des marqueurs acoustiques pentés par rapport au drain. À l'abscisse $55 \mathrm{~m}$, les différents trains d'ondes sont fortement atténués, indiquant la présence de fractures ouvertes. La figure 3.12 montre la section acoustique après filtrage des événements à vitesse apparente grande. Les événements obliques, associés à des réflexions sur des réflecteurs acoustiques, sont clairement visibles.

Les enregistrements du champ total fournis par les outils multi-émetteurs et multirécepteurs utilisés en diagraphie acoustique permettent de faire une micro-sismique dans le puits, basée sur l'analyse des modes réfléchis ou diffractés sur des discontinuités d'impédance acoustique internes aux formations ou sur des limites de formations. 

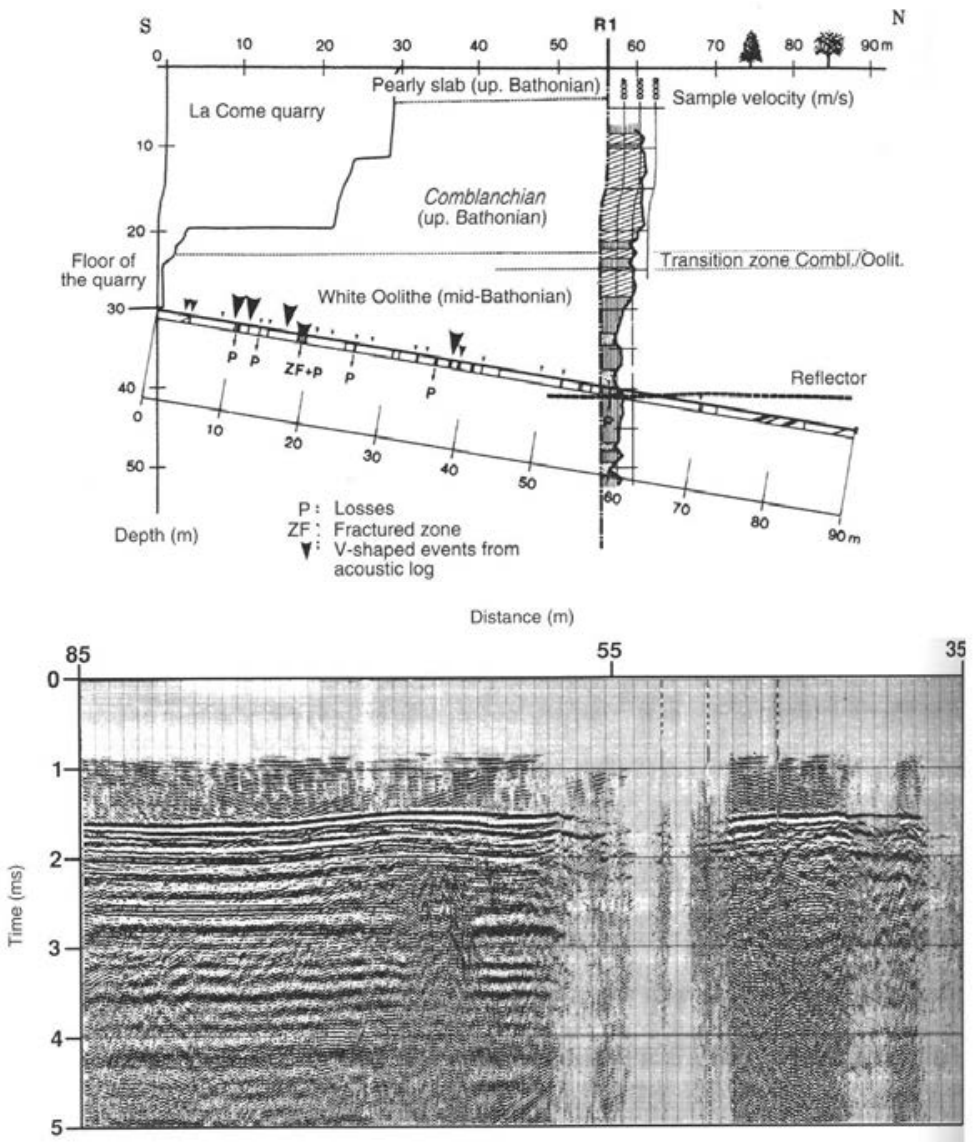

Figure 3.11 Imagerie acoustique en carrière (d'après Mari et al., 1994). En haut : coupe géologique de la carrière de Ravières. En bas : section acoustique à déport constant (3 m). Données brutes.

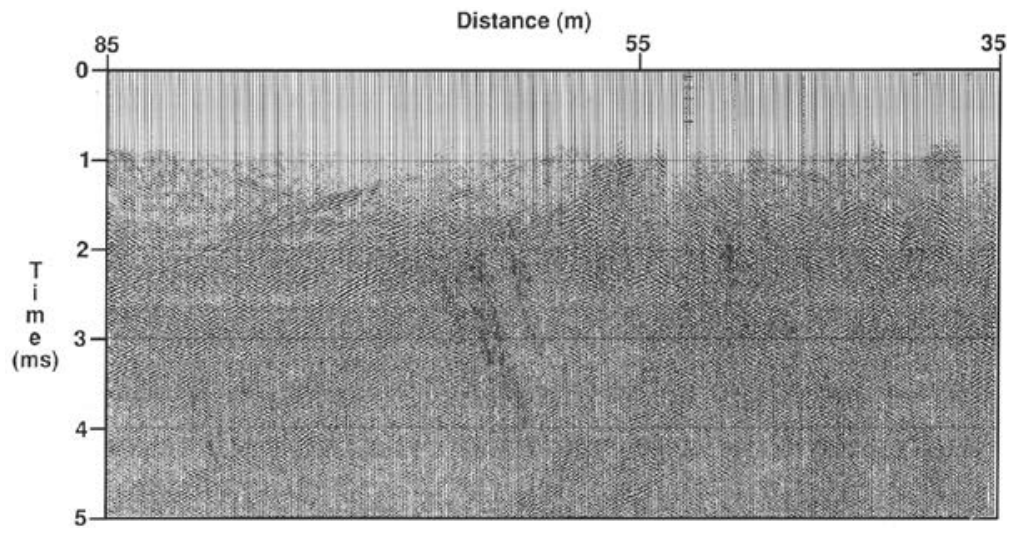

Figure 3.12 Imagerie acoustique en carrière : ondes réfléchies (d'après Mari et al., 1994). 
La figure 3.13 est un exemple d'imagerie par sections acoustiques obtenu dans un réservoir gréseux entrecoupé par des bancs de carbonates (Fortin et al., 1991). L'image acoustique met clairement en évidence la répartition des bancs de carbonates et leurs pendages par rapport au puits. Cette approche de l'estimation des pendages par acoustique nécessite un traitement complet de type couverture multiple.

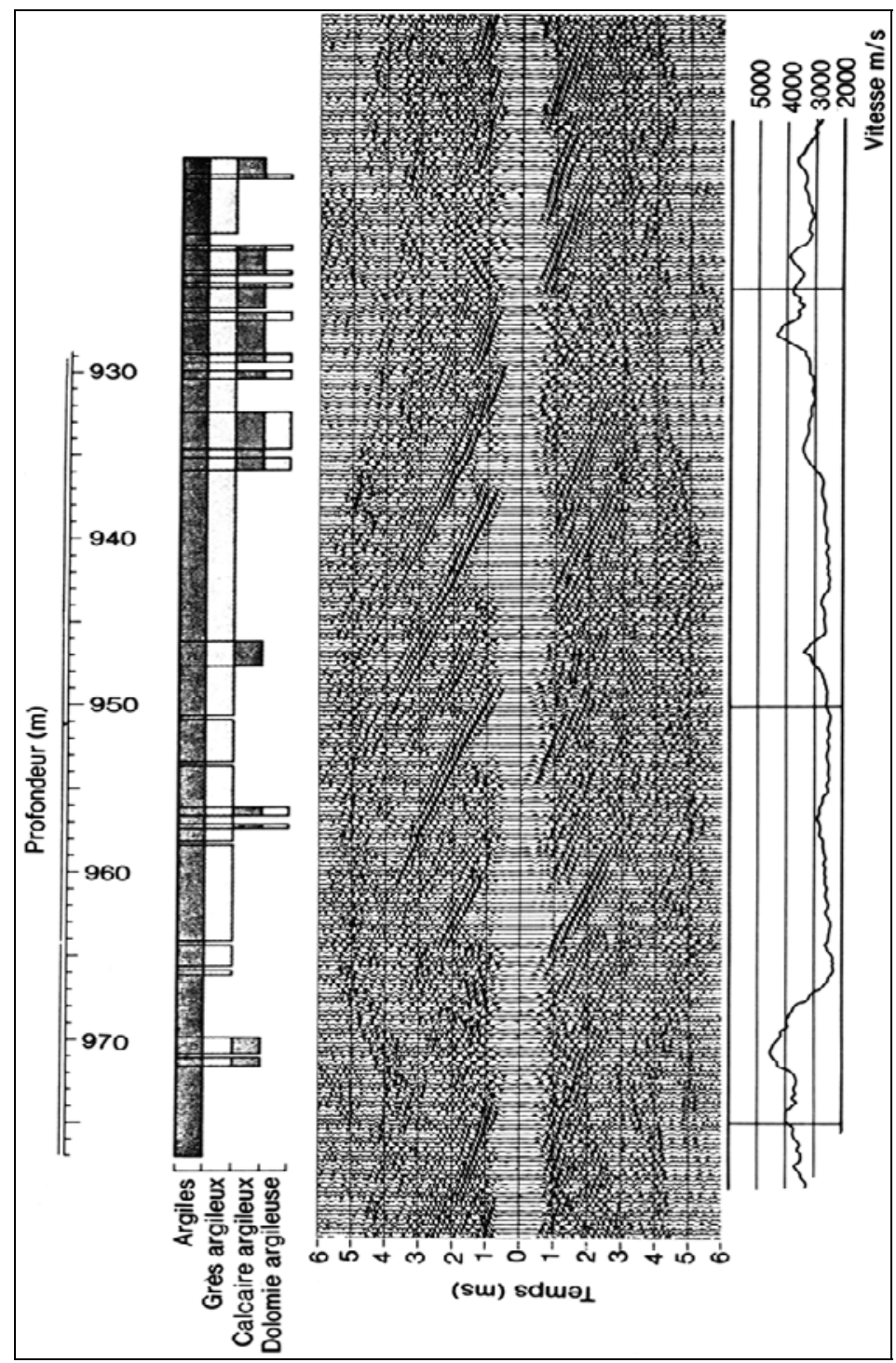

Figure 3.13 Imagerie de puits à l'aide de données acoustiques: ondes réfléchies. Outil EVA (SNEAP), traitement de type couverture multiple (CGG) (d'après Fortin et al., 1991). 


\subsection{Caractérisation d'une formation à l'aide des ondes de Stoneley}

Les ondes de Stoneley sont utilisées pour évaluer la lenteur $S$ des formations lentes, étudier la fracturation et accéder à une estimation de la perméabilité.

Les ondes de cisaillement ne peuvent être générées que si la vitesse $S$ de la formation est supérieure à la vitesse de l'onde de compression dans la boue ; la formation est alors dite rapide (contrairement aux formations dites lentes). En formation lente, la vitesse des ondes $S$ peut être estimée indirectement en puits non tubé à partir de l'équation de dispersion des ondes de Stoneley (Biot, 1956 ; Cheng et al., 1981).

Aux fréquences soniques (1-20 kHz), il est donc nécessaire de mesurer indépendamment six paramètres pour déduire de l'équation de dispersion des modes de Stoneley la vitesse $S$ de la formation. Ces paramètres sont : la vitesse de phase des ondes de Stoneley à une fréquence particulière, la densité du fluide, la densité de la formation, le diamètre du puits, la vitesse des ondes de compression dans la formation.

L'exemple suivant est un exemple de diagraphie acoustique enregistrée dans une formation lente, composée de marnes dans la partie supérieure et de calcaire dans la partie inférieure. La limite entre les marnes et les calcaires est située à $105 \mathrm{~m}$. Les données ont été acquises avec un outil monopole à trois récepteurs distants de $20 \mathrm{~cm}$. Le déport source - premier récepteur est de $60 \mathrm{~cm}$. La figure 3.14 montre à gauche les trois sections à déport constant enregistrées avec l'outil acoustique. Sur chaque section, on peut observer en première arrivée l'onde réfractée $\mathrm{P}$ de faible amplitude. L’onde réfractée $\mathrm{P}$ est suivie par une onde de très forte amplitude et basse fréquence qui est l'onde de Stoneley. La mesure des vitesses des ondes P et de Stoneley est faite par semblance. Le panneau de semblance est présenté à gauche des sections acoustiques. L'axe vertical représente la profondeur et l'axe horizontal représente la lenteur (inverse de la vitesse). La semblance est codée en couleur et exprimée en pourcent (en rouge les fortes valeurs). Le pointé des maxima de semblance (indiqué par les lignes noires continues) fournit pour chaque onde la valeur de la lenteur en fonction de la profondeur. Les logs de lenteur sont ensuite convertis en logs de vitesse (figure 3.14, droite). On peut noter un fort coefficient de corrélation entre les 2 logs de vitesse $(0,854)$. La fréquence dominante de l'onde de Stoneley est de $2 \mathrm{kHz}$. À ces basses fréquences, l'équation de dispersion de l'onde de Stoneley peut être approchée par une équation simplifiée proposée par White (1965). L'équation de White s'écrit :

$$
\frac{1}{V_{s t}^{2}}-\frac{1}{V_{f}^{2}}=\frac{\rho_{f}}{\rho} \cdot \frac{1}{V_{s}^{2}}
$$

avec $V_{s t}$ la vitesse de l'onde de Stoneley en basse fréquence, $V_{f}$ la vitesse du fluide de formation (ici l'eau), $V_{s}$ la vitesse $S$ de la formation, $\rho$ la densité de la formation, $\rho_{f}$ la densité du fluide. 

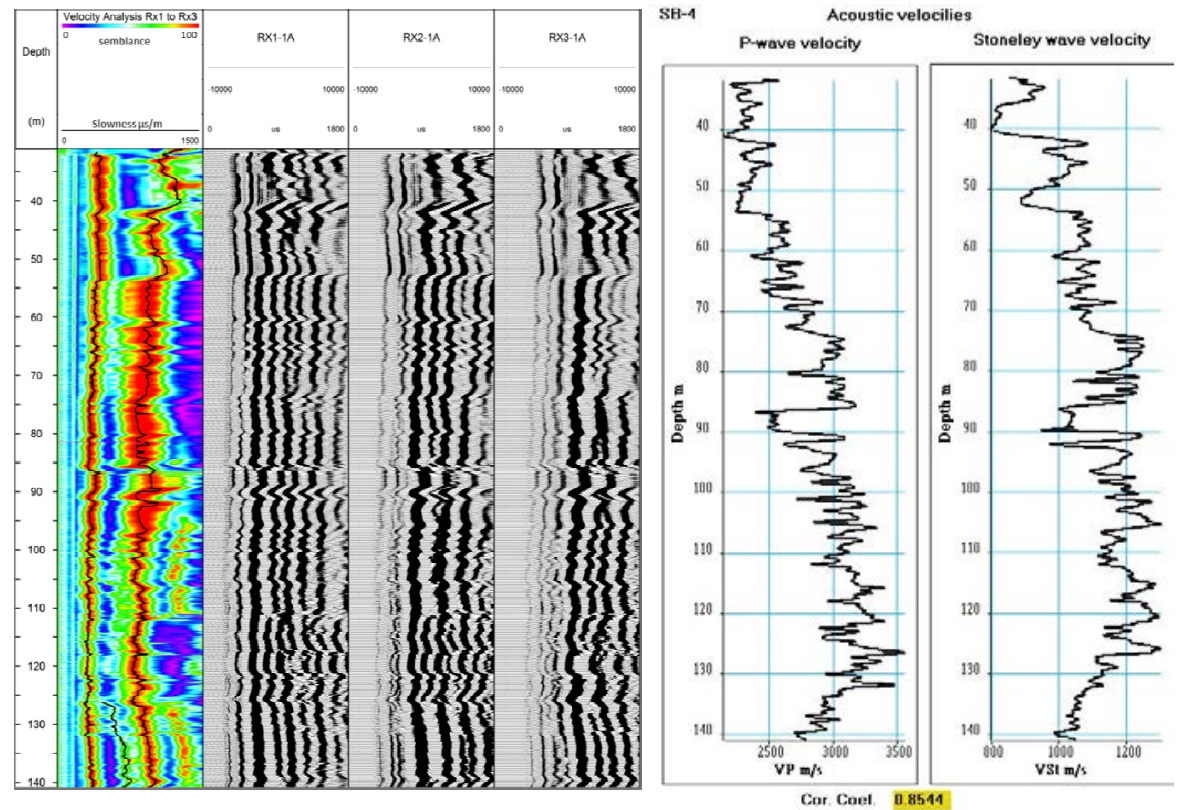

Figure 3.14 Diagraphie acoustique en formation lente. À gauche : panneau de vitesse et sections acoustiques à déport constants. À droite: logs de vitesse des ondes $P$ et des ondes de Stoneley.

Si le log de densité n’a pas été enregistré, ce qui est le cas dans l'exemple présenté, ce dernier peut être calculé à partir de la vitesse $V_{p}$ de la formation en utilisant la loi de Gardner :

$$
\rho=\alpha \times V_{p}^{\beta}
$$

Les équations de White et de Gardner sont utilisées simultanément pour ajuster les coefficient $\alpha$ et $\beta$ de la loi de Gardner et calculer la vitesse $V_{s}$ et la densité $\rho$ de la formation avec les contraintes suivantes :

1. la vitesse $S$ de la formation doit être inférieure à la vitesse de l'onde $\mathrm{P}$ dans le fluide ;

2. le coefficient de Poisson doit rester dans l'intervalle 0,3 et 0,5 , caractéristiques de marnes et de formations déconsolidées.

La figure 3.15 montre de gauche à droite : la densité Gardner, la vitesse $S$ estimée par la vitesse des ondes de Stoneley, le rapport $V_{p}$ sur $V_{s}$ et le coefficient de Poisson. 
SB-4
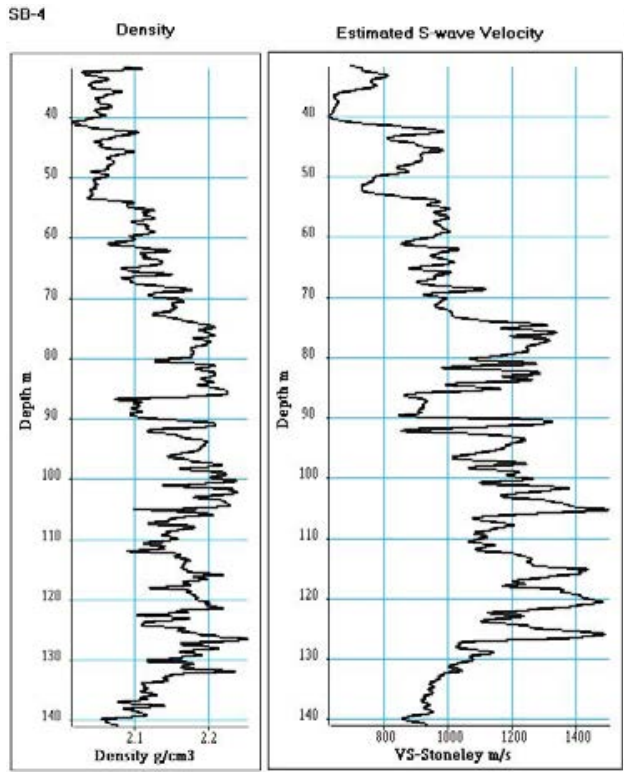

SB-4
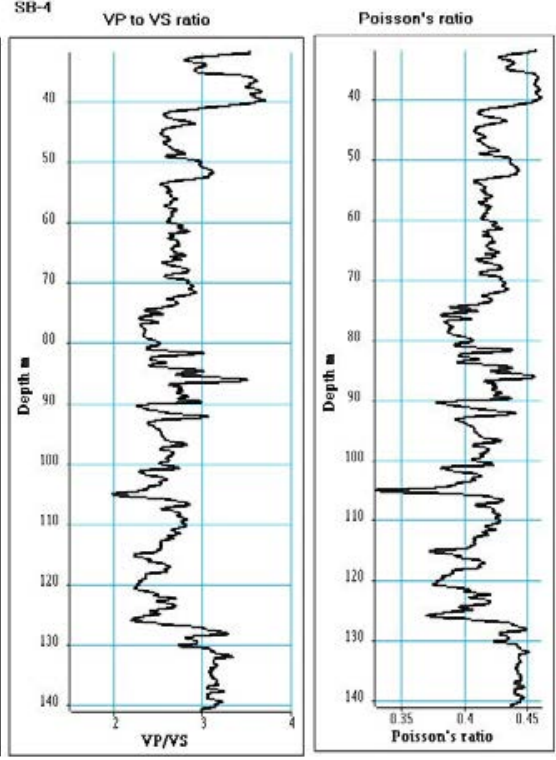

Figure 3.15 Logs acoustiques, de gauche à droite: densité, vitesse S estimée par la vitesse des ondes de Stoneley, rapport $V_{p}$ sur $V_{s}$ et coefficient de Poisson.

La réponse de l'onde de Stoneley est fortement liée à l'état de continuité de la paroi du puits. Sa transmission est guidée par l'interface eau ou boue contenue dans le forage et le mur de celui-ci. Elle est donc particulièrement affectée par la solution de continuité de la paroi du forage, et son exploitation ainsi que son traitement mettent en évidence la fracturation et le degré d'ouverture de celle-ci. L'atténuation des ondes de Stoneley (diminution d'amplitude et abaissement de la fréquence) est utilisée pour caractériser le milieu fissuré. De plus, on observe des phénomènes de conversion d'ondes aux limites des zones fracturées. Ces phénomènes sont très marqués sur les ondes de Stoneley, surtout en présence de fractures ouvertes.

\subsection{Conclusion}

Par rapport aux autres diagraphies, la diagraphie acoustique a une résolution verticale équivalente, mais un rapport investigation latérale sur résolution verticale très nettement supérieur. La diagraphie acoustique a une investigation latérale de quelques centimètres pour les modes d'interface, décimétrique à métrique pour les modes rétractés, et de quelques mètres à la dizaine de mètres pour les modes réfléchis. 
La diagraphie acoustique est principalement utilisée pour :

- mesurer les vitesses des formations (en compression et cisaillement) et calculer les modules d'élasticité (mesure dynamique $2-40 \mathrm{kHz}$ ) ;

- établir la relation temps-profondeur très haute résolution par intégration de la courbe des lenteurs (inverse de la vitesse) ;

- réaliser des films synthétiques pour caler la sismique réflexion de surface (voir chapitre 4) ;

- mesurer l'atténuation et l'anisotropie (mode dipôle) d'une formation ;

- identifier la lithologie en combinaison avec d'autres diagraphies ;

- étudier la fracturation et détecter des hétérogénéités ;

- évaluer la cimentation d'un tubage (voir chapitres 1 et 4) ;

- évaluer la porosité et appréhender la perméabilité ;

- mesurer les pendages ;

- fournir une micro-sismique de détail (ondes réfléchies) au voisinage du puits ; opérations à privilégier dans les puits fortement déviés ou horizontaux.

Il faut aussi prendre en considération les éléments suivants :

- La mesure acoustique doit être faite dans un puits rempli d'eau (boue). Il est préférable de travailler en trou ouvert (voire tubé PVC). Il est souhaitable de faire une mesure continue des diamètres du forage (diagraphie : diamétreur) pour détecter les zones cavées. L'outil doit être centré par des centreurs lors des mesures acoustiques.

- La vitesse de logging doit être faible (4 à $6 \mathrm{~m} / \mathrm{min}$ ) pour respecter les conditions d'échantillonnage en distance et pour éviter les bruits de raclement créés par les centreurs. En général, un filtre en fréquence (coupe bas : $1 \mathrm{kHz}$ ) est utilisé à l'acquisition pour filtrer ces bruits.

- La mesure acoustique peut être réalisée en trou tubé acier, lorsque les tubages sont parfaitement cimentés. La présence de phénomènes de résonance dus à la mauvaise cimentation est utilisée pour évaluer la cimentation (log de cimentation).

- Les conditions de puits, en cas de puits tubé mal cimenté, peuvent rendre les mesures difficiles. Les ondes associées aux vibrations du casing doivent être filtrées. Ce traitement ne permet pas toujours d'extraire les ondes de volume caractéristiques de la formation et de mesurer les paramètres de formation.

- La mesure acoustique est favorisée par l'utilisation d'outils longs (3 à 4 m entre l'émetteur et les récepteurs). La mise en œuvre peut receler quelques difficultés lorsque le mât du système de levage n'est pas suffisamment haut. Dans ce cas, l'outil, s'il est souple, peut être introduit dans le puits en le courbant. L'outil peut aussi être introduit par éléments constitutifs connectables entre eux. 


\section{Références}

Arditty P.C., Arens G., Staron P., 1984, Improvements of formation properties and evaluation through processing and interpretation results of the EVA tool records. SEG 54th Annual Meeting expanded abstracts.

Biot M.A., 1956, Theory of propagation of elastic waves in a fluid-saturated porous solid: I - Low waves in a fluid-saturated porous solid: I - Low frequency range; II - Higher frequency range. The Journal of the Acoustical Society of America, 28, 2, pp. $168-178$ et $179-191$.

Cheng C.H., Toksöz M.N., 1981, Elastic wave propagation in a fluid-filled borehole and synthetic acoustic logs. Geophysics, 46, 1042-1053.

Coppens F., Mari J.L., 1995, Application of the intercept time method to fullwave form acoustic data. First Break, 13: 11-20.

Fortin J.P., Rehbinder N., Staron P., 1991, Reflection imaging around a well with the Eva full-waveform tool. The Log Analyst, 32, 3, 271-278.

Gardner G.H.F., Gardner L.W., Gregory A.R., 1974, Formation velocity and density. The diagnostic basis for stratigraphic traps. Geophysics, 39, 770-780.

Gaudiani P., 1982, Acoustic coring in granite with a small diameter logging tool. Proceedings, Workshop about geophysical investigations in connection with geological disposal of radioactive waste, Nuclear Energy Agency Organization for Economic Cooperation and Development, Ottawa: 157-171.

Kaneko F., Kanemori T., Tonouchi K., 1990, Low-Frequency Shear Wave Logging in Unconsolidated Formations for Geotechnical Applications. Geophysical Applications for Geotechnical Investigation, ASTM STP 1101, F.L. Paillet and W.R. Saunders, Eds. American Society for Testing and Materials, Philadelphia, pp. 79-98.

Mari J.L., Gavin P., Coppens F., Wicquart E., 1992, Traitement des diagraphies acoustiques. Éditions Technip, Paris.

Mari J.L., Gavin P., Coppens F., 1994, An example of acoustics and very high resolution seismic in a near-horizontal borehole. First Break, 12, 21-29.

Mari J.L, Arens G., Chapellier D., Gaudiani P., 1998, Géophysique de gisement et de génie civil. Éditions Technip, Paris.

Mari J.L, Gaudiani P., Delay J., 2011, Characterization of geological formations by physical parameters obtained through full waveform acoustic logging, Physics and Chemistry of the Earth, 36, 1438-1449, Elsevier Ltd.

Mari J.L., 2015, Signal processing for geologists \& geophysicists, e-book, DOI:10.2516/ ifpen/2011002, http://books.ifpenergiesnouvelles.fr/ebooks/signal-processing/

Mari J.L., Porel G., 2015, Automated karstic reservoir analysis utilizing attributes, We N117 02, 77th EAGE Conference \& Exhibition, Madrid IFEMA, Spain, 1-4 June. 
Morris C.F., Little P.M., Letton W., 1984, A new sonic array tool for full waveform logging, 59th Annual Conference of the Society of Petroleum Engineers of AIME, Houston, Texas, expanded abstracts, pp. 16-19.

Paillet F.L., Turpening R., 1984, Borehole and surface to borehole seismic applications in fracture characterization. SEG 54th Annual Meeting expanded abstracts.

Quiblier J., 1997, Propagation des ondes en géophysique et en géotechnique. Modélisation par méthodes de Fourier. Éditions Technip, Paris.

Rosenbaum J.H., 1974, Synthetic micro-seismograms logging in porous formation. Geophysics, 39, 14-32.

Summers G.C., Broding R.A., 1952, Continuous Velocity Logging. Geophysics, 17, 598-614.

Vogel C.B., 1952, A seismic logging method. Geophysics, 17, 579-586.

White J.E., 1965, Seismic waves: Radiation, transmission and attenuation. McGrawHill Book Company, New York.

Zemanek J., Williams D.M., Schmitt D.P., 1991, Shear-wave logging using multipole sources, The $\log$ Analyst, 32, 3, 233-241. 
